\title{
Bioreactor Development for Lung Tissue Engineering
}

\author{
Angela Panoskaltsis-Mortari
}

Published online: 6 February 2015

(C) Springer International Publishing AG 2015

\begin{abstract}
Rationale Much recent interest in lung bioengineering by pulmonary investigators, industry, and the organ transplant field has seen a rapid growth of bioreactor development ranging from the microfluidic scale to the human-sized whole lung systems. Comprehension of the findings from these models is needed to provide the basis for further bioreactor development.

Objective The goal was to comprehensively review the current state of bioreactor development for the lung.

Methods A search using PubMed was done for published, peer-reviewed papers using the keywords "lung" AND "bioreactor" or "bioengineering" or "tissue engineering" or "ex vivo perfusion".

Main Results Many new bioreactors ranging from the microfluidic scale to the human-sized whole lung systems have been developed by both academic and commercial entities. Microfluidic, lung-mimic, and lung slice cultures have the advantages of cost-efficiency and high throughput analyses ideal for pharmaceutical and toxicity studies. Perfused/ ventilated rodent whole lung systems can be adapted for mid-throughput studies of lung stem/progenitor cell development, cell behavior, understanding and treating lung injury, and for preliminary work that can be translated to human lung bioengineering. Human-sized ex vivo whole lung bioreactors incorporating perfusion and ventilation are amenable to automation and have been used for whole lung decellularization and recellularization. Clinical scale ex vivo lung perfusion
\end{abstract}

This article is part of the Topical Collection on Tissue Engineering and Regeneration

A. Panoskaltsis-Mortari $(\square)$

Departments of Pediatrics and Medicine; Blood and Marrow

Transplant Program; Pulmonary, Allergy, Critical Care and Sleep

Medicine, University of Minnesota, MMC 366, 420 Delaware St.

SE, Minneapolis, MN 55455, USA

e-mail:panos001@umn.edu systems have been developed for lung preservation and reconditioning and are currently being evaluated in clinical trials.

Conclusions Significant advances in bioreactors for lung engineering have been made at both the microfluidic and the macroscale. The most advanced are closed systems that incorporate pressure-controlled perfusion and ventilation and are amenable to automation. Ex vivo lung perfusion systems have advanced to clinical trials for lung preservation and reconditioning. The biggest challenges that lie ahead for lung bioengineering can only be overcome by future advances in technology that solve the problems of cell production and tissue incorporation.

Keywords Bioreactor $\cdot$ Bioengineering $\cdot$ Tissue Engineering $\cdot$ Lung

\section{Introduction}

The fast-emerging field of lung bioreactor development has been spurred by three main incentives: (1) the need for costefficient, higher throughput studies for drug discovery, (2) the desire to study lung stem cells and regenerative strategies in a controllable, physiologic context, and (3) the transplant organ shortage driving the bioengineering of new lungs or preserving and reconditioning lungs that do not meet the criteria for transplantation.

The term "bioreactor" can encompass any system that can support biological activity. With respect to the lung, the range includes 2-dimensional tissue culture to whole lung preservation systems. This review will not cover traditional 2dimensional lung cell cultures or air-liquid interfaces. The reader is referred to recent reviews on these topics $[1 \bullet]$. 


\section{Methods}

A search using PubMed was done for published, peerreviewed papers using the keywords "lung" AND "bioreactor" or "bioengineering" or "tissue engineering" or "ex vivo perfusion". For this review, no specific inclusion or exclusion criteria were determined a priori.

\section{Results}

Lung Mimics

Small scale bioreactors have been developed to approximate the lung environment more accurately than simple, static airliquid interface cultures. Their best uses are to study lung cell behavior in drug discovery and as pharmacokinetic models to study drug toxicities. In early work, hollow fiber bioreactors proved capable of maintaining human airway glandular cells and lung cancer cells in the context of culture medium perfusion $[2,3]$. In other work, lung epithelial cells exposed to controlled airflow in semipermeable microfiber cultures have resulted in confluent monolayers capable of forming tight junctions [4]. This bioreactor concept has been advanced further by the use of microfabrication techniques to fabricate the equivalents of microvasculature and airway parenchyma to simulate the gas exchange apparatus [5].

The advent of microfluidic and chip devices has brought high throughput systems ideal for pharmacokinetic and toxicity studies. These devices incorporate perfusion and mechanical strain to simulate the stretch of breathing and allow for the interaction of different cell types to mimic the complex environment [6, 7•] (Fig. 1).
Even simpler approaches have been used to approximate the 3-dimensional lung environment. In one system, lung epithelial cells were cultured in 3-D biodegradable polymer gels that were expanded using a mechanical actuator to create the expansion normally experienced by the alveolar region of the lung during inspiration and exhalation [8]. The system fit into standard 6-well plates and was continually perfused with culture media, although the perfusion was into the wells and not directly through the tissue construct. Others have used lung slices or small pieces of lungs in tissue culture plates onto which cells are seeded. To some extent the cells can migrate through the lung matrix $[9 \cdot \bullet]$, or be coaxed by centrifugation $[10 \bullet \cdot$.

Another aspect of lung bioengineering is the development of ambulatory lung assist devices using microfluidic vascular networks to mimic alveolar-capillary membranes [11-15]. Another miniaturized device using stacked, microfluidic oxygenator units may have potential to treat respiratoryinsufficient newborns and is currently being tested in piglets $[16 \bullet]$.

\section{Large Airway Bioreactors}

For upper airways, (trachea, bronchus), horizontal and upright bioreactors have been developed. An upright system was developed by the Niklason lab and utilized pulsating air flow, causing radial distension that provided mechanical stimulation to the bioengineered bronchiole [17] (Fig. 2a). The horizontal system used by the Macchiarini and Birchall labs in the clinical setting (compassionate use basis) was developed by Harvard Apparatus with Hugo Sachs Elecktronik and has been outfitted for fluid flow through and around the bioengineered graft, but without the use of external pumps, allowing for
A
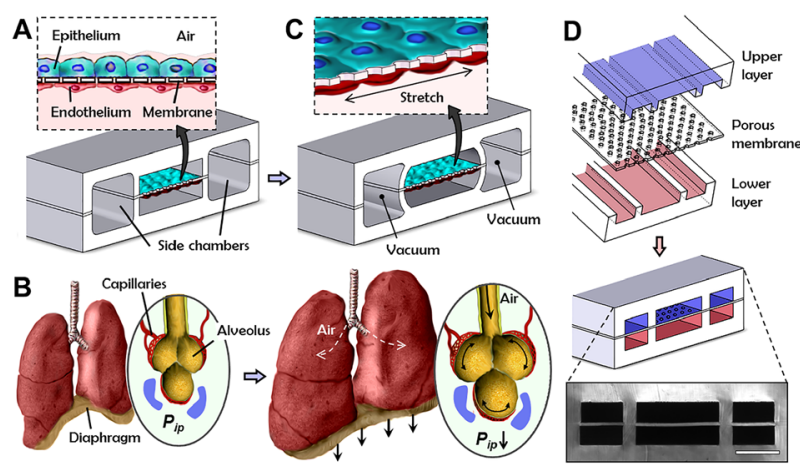

Fig. 1 Lung-Mimic Bioreactors. a) "Lung-on-a-chip" microfluidic device developed by the Ingber lab (From Huh D, Matthews BD, Mammoto A et al. Reconstituting organ-level lung functions on a chip. Science 2010; 328:1662-8. Reprinted with permission from AAAS) [6]. b) Microfluidic device developed by the Nichols/Cortiella lab (From
B
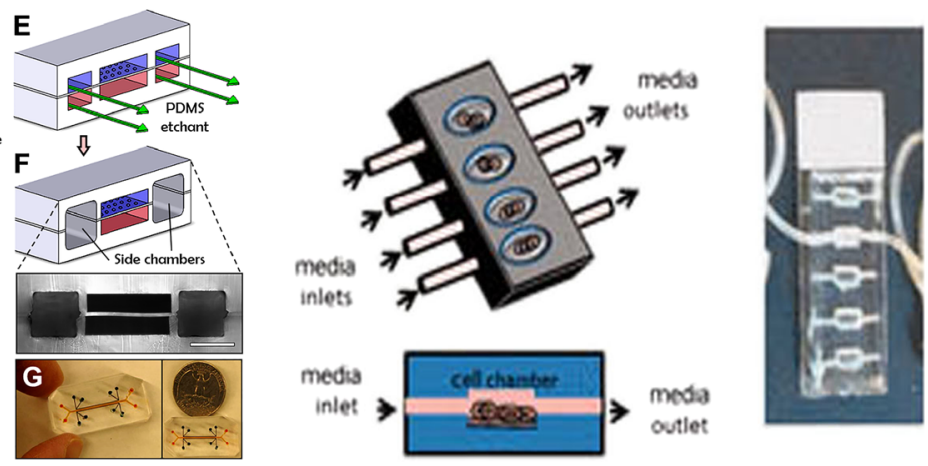

Nichols JE, Niles JA, Vega SP et al. Modeling the lung: Design and development of tissue engineered macro- and micro-physiologic lung models for research use. Exp Biol Med (Maywood) 2014; 239:1135-69. Reprinted by Permission of SAGE) [1•] 


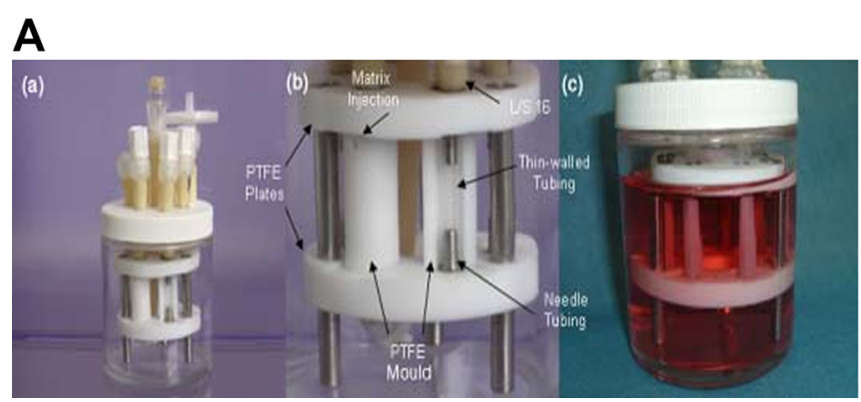

Fig. 2 Large Airway Bioreactors. a) Device developed in a vertical orientation by the Niklason lab (From Miller C, George S, Niklason L. Developing a tissue-engineered model of the human bronchiole. J Tissue Eng Regen Med 2010; 4:619-27; with permission) [17]. b) Commercial horizontal rotating device (Harvard Apparatus Regenerative

easier GMP adaptation [18] (Fig. 2b). The decellularized or bioengineered airways used clinically are only briefly incubated with autologous cells and, thus, are not totally recellularized when transplanted into the patient. This pioneered the strategy of using the human body itself as the bioreactor. The Delaere group has pushed this "human body as bioreactor" concept further by bioengineering the allogeneic donor trachea first within the forearm of the recipient and then moving the allograft to replace the damaged trachea $[19$, $20 \bullet \cdot$.

\section{Whole Lung Bioreactors}

The breakthrough that catapulted whole organ bioengineering was the ability to decellularize an intact organ by perfusion resulting in intact acellular scaffolds that retained vascular conduits. For bioengineering using intact lung scaffolds, bioreactors have been developed for decellularization and, by design, are also amenable for recellularization so as to maintain a sterile, closed system combined with perfusion and ventilation. Comparisons of decellularization solutions have been reviewed elsewhere $[21 \cdot, 22,23]$.

In order to recellularize both vascular and airways conduits, a bioreactor must include independent access lines with integrated pressure transducers to enable flow/volume-based control of pressure, among other requirements (Table 1). Pulsatile, instead of continuous, perfusion though the vasculature will simulate heart-driven blood flow and needs to appropriately distribute nutrients while removing cellular and extracellular waste products. It should also provide mechanical ventilation, ideally by negative pressure to avoid lung damage, but positive pressure inflation may also be needed to reverse lung collapse and allow for airway recruitment. Some bioreactors have been used for whole lungs that are already decellularized and have integrated cell reseeding. These range from rodentsized [24-27] to primate-sized [28••] (Fig. 3a-c). These bioreactors are used in incubators to maintain physiologic temperature. There has not been consistency as to the incorporation

\section{B}

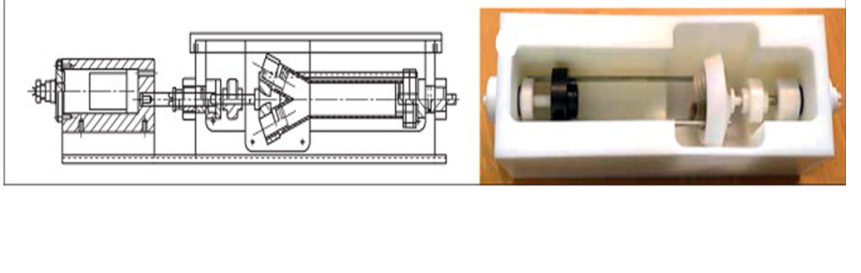

Technologies-HART) used for human transplants by Macchiarini and Birchall groups (Reprinted from Jungebluth P, Alici E, Baiguera S et al. Tracheobronchial transplantation with a stem-cell-seeded bioartificial nanocomposite: a proof-of-concept study. Lancet 2011; 378:1997-2004, with permission from Elsevier) [18]

of vascular perfusion and airway ventilation. Some systems are ventilation only [26] (Fig. 3a) while others have developed simple closed system bioreactors for rodent lungs that have vascular perfusion but not ventilation [29] (Fig. 3b). It has been shown that ventilation motions alone can allow for passive nutrient perfusion of the pulmonary vasculature, i.e., vascular perfusion is not required. To maintain a native, normal lung, vascular perfusion alone was not sufficient [25]; airway ventilation with nutrient medium was required as ventilation with air resulted in loss of epithelium. Despite the lack of ventilation motion, lung cancer cells grew quite well in this system, developing complex tumor nodules indicating that bioreactor requirements for lung cancer models may not be as complex as they are for normal lung tissue. Others have used a commercially available bioreactor for rodents that maintains physiologic temperature with a water-jacketed chamber that holds the lungs [30] (Fig. 3d and e top). The apparatus is used for reseeding and monitoring of function $\left(\mathrm{pO}_{2}, \mathrm{pCO}_{2}, \mathrm{pH}, \mathrm{PFTs}\right)$. The temperature of the perfusate is maintained with a heating coil and the lungs are ventilated with heated, humidified air. Also to be considered is whether and when to use liquid versus air ventilation and when that transition should take place, simulating the fetal to post-natal environment. The ventilation strategy (frequency, volume, deep inspiration for recruitment, variable stretch) to be

Table 1 Lung Bioreactor Requirements

1. Accommodate cell-seeding for all the cell types through either airway
or vasculature
2. Provide nutrients
3. Remove waste/toxic products
4. Provide and maintain proper blood gases
5. Provide ventilation at appropriate frequencies
6. Monitor airway pressures
7. Monitor and maintain physiologic vascular pressure
8. Maintain sterility
9. GMPable


A Ventilation bioreactor
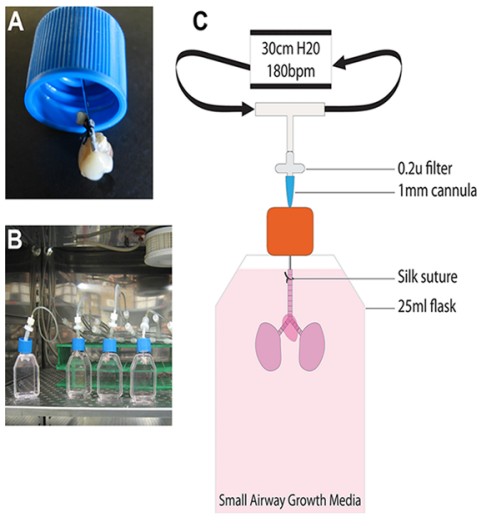

D

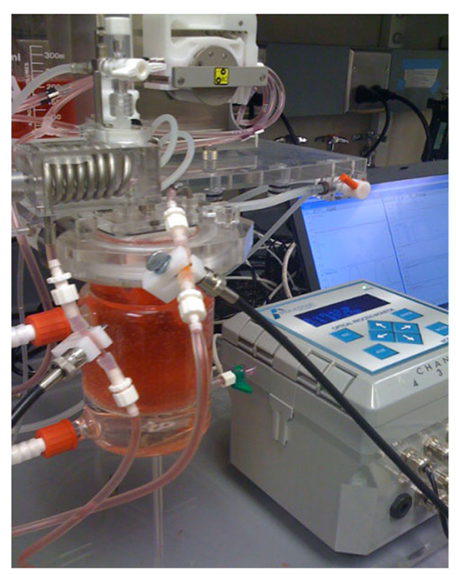

B

$$
\text { Perfusion bioreactor }
$$



E

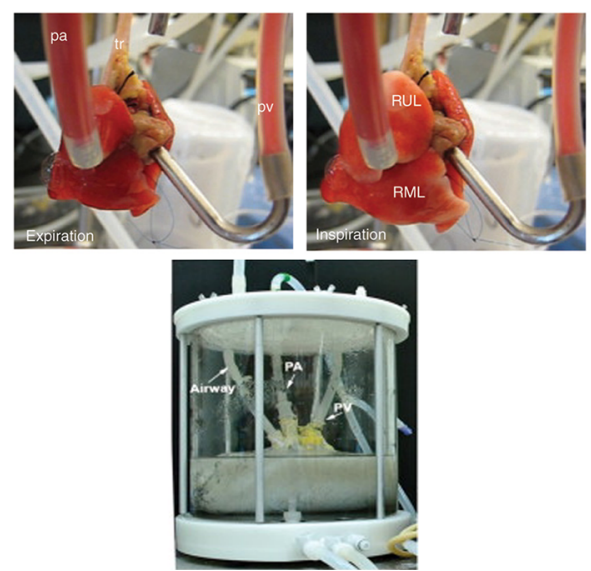

C

\section{Perfusion/ventilation}



$\mathbf{F}$

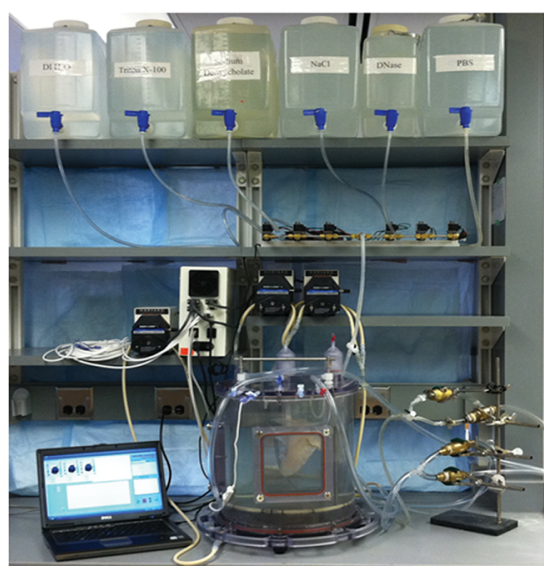

Fig. 3 Whole Lung Bioreactors. a) Simple ventilation-only bioreactor for cell reseeding developed by the Panoskaltsis-Mortari lab [26]. Multiple units can be set up in parallel. b) Perfusion-only bioreactor from the Kim group developed for decellularization and recellularization that is also amenable to multi-unit set-up (Reprinted from Mishra DK, Thrall MJ, Baird BN et al. Human lung cancer cells grown on acellular rat lung matrix create perfusable tumor nodules. Ann Thorac Surg 2012; 93:1075-81, with permission from Elsevier) [29]. c) Whole lung bioreactor developed for non-human primate lung decellularization and recellularization that incorporates both ventilation and perfusion developed by the Bunnell group (From onvillain RW, Scarritt ME, Pashos NC et al. Nonhuman primate lung decellularization and recellularization using a specialized large-organ bioreactor. J Vis Exp 2013:e50825, with permission from JoVE) [28••]. This is a scaled-up version of the rodent bioreactor developed by the Niklason group [25]. d) Commercially available (HART) ventilation/perfusion rodent

used will likely be dependent on the stage of the bioengineered lung with respect to cell types incorporated, maturity, and mechanical properties.

For human-sized lungs, a custom-designed chamber for decellularization with a pressure-controlled pump system was recently reported [9••] (Fig. 3e, bottom). Decellularization was considered "complete" by visual inspection. Cells were allowed to seed the lungs through the airway by gravity. For decellularization, several groups have used the isolated lung perfusion system that has gone through bioreactor that can be used for recellularization and functional assessment showing integration of $\mathrm{O}_{2}$ and $\mathrm{CO}_{2}$ sensors connected to gas measurement unit (photo courtesy of the author Angela Panoskaltsis-Mortari). e) Rodent (top) [30] and human (bottom) [9••] closed-system perfusion and ventilation bioreactors developed by the Ott lab showing detail of vascular and airway cannulations. (Adapted by permission from Macmillan Publishers Ltd: Ott HC, Clippinger B, Conrad $\mathrm{C}$ et al. Regeneration and orthotopic transplantation of a bioartificial lung. Nat Med 2010; 16:927-33) [30]; and (Reprinted from Gilpin SE, Guyette JP, Gonzalez G et al. Perfusion decellularization of human and porcine lungs: Bringing the matrix to clinical scale. J Heart Lung Transplant 2013, with permission from Elsevier) [9・•]. f) Automated human-sized decellularization/recellularization system developed by the Panoskaltsis-Mortari lab using commercial chamber (HART) and customized computer-controlled valve-manifold assembly $[31 \bullet \bullet]$.

several iterations and is now known as the Organ Regenerative Control Acquisition bioreactor (commercially available from Harvard Apparatus Regenerative Technologies, HART)[10••, 31••, 32]. Decellularization times vary, but can be standardized by automation [31••] (Fig. 3f). Although others have found that decellularization time can be decreased substantially by increasing perfusion pressure [32], structural integrity of the distal lung appears to be compromised. There is no consensus on what the ideal time for decellularization should be, as it is likely also dependent on the solutions used, 
but scaffold integrity cannot be compromised in exchange for speed using supraphysiologic pressures that can damage the fragile microvascular areas. Some investigative groups use only vascular perfusion for decellularization, but a comparison of using both vascular and airway conduits, versus using each alone, showed that using both accesses is more efficient [26].

\section{Evaluation of ex Vivo Perfused Lung Constructs}

There are several methods to assess the mechanical properties of bioengineered lungs, and most of them require tissue sampling to evaluate stiffness and failure properties [33•]. This would be invasive at the bioreactor level, but would be useful in experiments aimed at determining standardization criteria. At the whole organ level, however, simpler pressure-volumeflow measurements using mechanical ventilation can provide resistance, compliance, and elastance of the lungs to ensure these are within physiologic range. Such measurements have already been incorporated into whole lung bioreactors. Although cardiac-induced lung motion has been considered in breathing models, the displacement is relatively small, and the costs/efforts of incorporating this parameter would not be justified at this early stage of bioreactor development [34].

Preservation of airway and vascular conduits has been monitored noninvasively using thermography [35•].
Alternatively, vascular permeability in real time may be determined using fluorophores [36•]. Although in-line sensors of $\mathrm{pO} 2$, pCO2, and $\mathrm{pH}$ have been incorporated using commercially available components (Fig. 3d), other functional information about cell status (glucose, lactate, electrolytes) will be required in real-time. Also not developed is the ability to assess formation of tight junctions and to measure transepithelial resistance. Further development of microsensors is needed.

Ex vivo lung perfused (EVLP) systems have been used for studying lung disease and potential therapeutic interventions $[37,38 \cdot]$. Many investigators do not use these systems for prolonged lung maintenance nor for bioengineering purposes. Rodent- sized systems have been available for several years (Harvard Apparatus), and human- sized chambers are also now available. Following the first demonstration of functional assessment of a human lung ex vivo [39], EVLP systems have now evolved into lung preservation systems [40] (e.g., Organ Care System from Transmedics ${ }^{\mathrm{TM}}$, and XPS ${ }^{\mathrm{TM}}$ from XVIVO Perfusion) and are currently being evaluated in clinical trials of lung transplantation for their ability to prolong organ preservation and to recondition lungs that would normally be turned down for transplant [41, 42] (Fig. 4). The clinical grade EVLP systems keep the lungs oriented in a horizontal position laying down on a solid surface, whereas the decellularization/ recellularization chambers discussed above hold the lungs upright, suspended in medium so as to minimize the mechanical
A

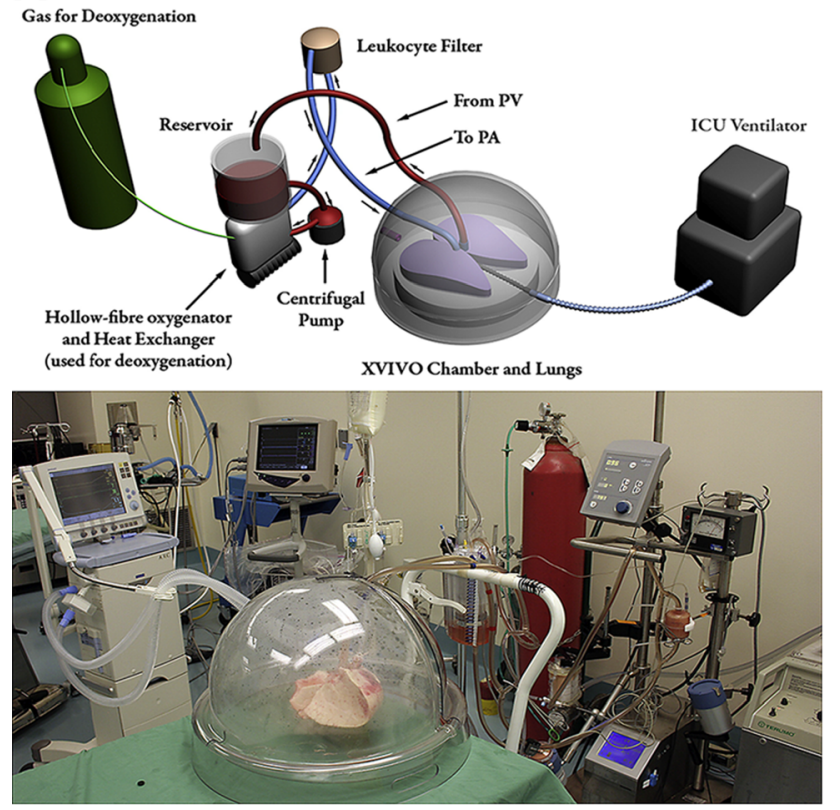

Fig. 4 Clinical Ex Vivo Lung Preservation Systems. a) "Dome" XVIVO $\mathrm{XPS}^{\mathrm{TM}}$ unit originally developed by the Keshavjee lab (Reprinted from Yeung JC, Cypel M, Waddell TK et al. Update on donor assessment, resuscitation and acceptance criteria, including novel techniques - Nonheart-beating donor lung retrieval and ex vivo donor lung perfusion. Thorac Surg Clin 2009; 19:261-74, with permission from Elsevier)
B

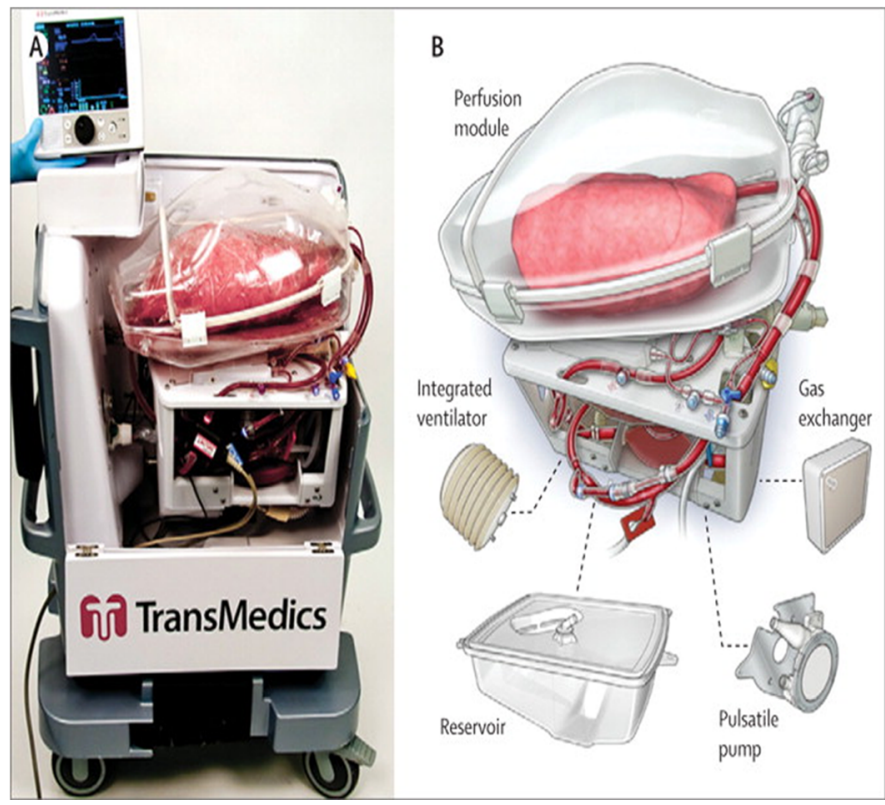

[56]. b) Organ Care Lung System (OCS ${ }^{\mathrm{TM}}$ by TransMedics) used by Warnecke et al. (Reprinted from Warnecke G, Moradiellos J, Tudorache I et al. Normothermic perfusion of donor lungs for preservation and assessment with the Organ Care System Lung before bilateral transplantation: a pilot study of 12 patients. Lancet 2012; 380:1851-8, with permission from Elsevier) [42] 
strain of the lung weighted down by the fluids in the vasculature and the airways, especially in the acellular state when the lung is atelectatic.

\section{Auxiliary Bioreactors}

A major challenge in bioengineering lung tissue large enough for human transplantation purposes will be the generation of sufficient numbers of cells to repopulate the acellular scaffolds. The generally accepted idea by most investigators studying lung bioengineering is to use the patient's own cells or at least HLA-matched cells. Some of the required cells could be easily expanded from the patient's bone marrow or blood, such as mesenchymal stromal cells or endothelial cells. However, expansion of epithelial cells will require other technologies including deriving them from reprogrammed iPS cells. Large-scale expansion has been achieved using controlled, stirred tank bioreactors for iPS and embryonic stem cells [43]. Automated, stirred bioreactors that can control for $\mathrm{pH}, \mathrm{pO}$, and temperature have been developed for stem cell expansion (Dasgip, Julich, Germany). Specific to the lung, others have achieved high efficiency of alveolar epithelial cell differentiation from ES cells using hydrogel encapsulation and rotating vessel bioreactors that can be automated and scaled up [44]. Given the recent advancements in knowledge about lung development, lung stem/progenitor cells, and their derivation from pluripotent stem cells $[45 \bullet, 46 \bullet, 47 \bullet, 48 \bullet$, 49••], further work is required in growing and differentiating the required cells to clinical scale. A rotating bioreactor that alternatingly exposes cells to air and liquid has been recently shown to support expansion of alveolar epithelial cells [50]. Although the clinical experience with bioengineered trachea and bronchus indicates that total re-epithelialization of those large airways may not be required prior to implantation, the distal areas of the lung need to be complete both on the endothelial side to avoid thrombogenicity and on the epithelial side for efficient gas exchange as well as to avoid fibroproliferative remodeling of a denuded basement membrane. It is not yet clear whether it is best to repopulate the lung with the entire composite of cells (stem/progenitor cells and fully differentiated cells) or to seed it with a smaller population consisting of stem/progenitors that will expand and differentiate into the required cell types being guided by the acellular matrix scaffold $[51,52 \bullet \cdot]$. The strategy to be used will also dictate what parameters and features will be needed in the bioreactor. The stem cell infusion process itself must be considered since cells respond poorly to shear stress especially if syringes are used [53]. Cell seeding volume and time can affect seeding efficiency and viability [54]. Ventilation protocols must be tailored so as to maintain as much of the lung expanded as possible without causing damage by overdistension [55]. This will likely vary depending on the bioengineering stage of the lung as it is "assembled" by sequential cell seeding in the bioreactor.

\section{Conclusions}

Lung bioreactor development has been expanding in the last five years as a result of targeted NIH funds, as well as commercial interests, in the need for developing more physiologic 3-D lung systems for identifying new treatment strategies, and providing transplantable lungs for devastating lung diseases. Significant advances have been made at both the microfluidic and the macroscale. The most advanced are closed systems that incorporate pressure-controlled perfusion and ventilation and are amenable to automation, having advanced to the clinical setting. Future efforts should be aimed at merging the most promising features of several systems and incorporating more real-time outputs of lung status and function, always keeping clinical translatability in mind.

Acknowledgments The author apologizes to investigators whose work was not cited in this review due to space limitations. However, many of those papers are cited in the reviews referenced here. The author thanks Ms. Kelsey Vigoren and Miss Marisa Mortari for help in preparing the manuscript. APM is partly funded by NHLBI R01HL108627 ("Overcoming Barriers to Bioengineering 3D Human Lung").

\section{Compliance With Ethics Guidelines}

Conflict of Interest The computer program code for an automated lung bioreactor valve-control system is available for licensing from the University of Minnesota to commercial entities, but it is free and opensourced for all academic investigators. Angela Panoskaltsis-Mortari is eligible to receive one ninth of any licensing fees.

Human and Animal Rights and Informed Consent This article does not contain any studies with human or animal subjects performed by any of the authors.

\section{References}

Papers of particular interest, published recently, have been highlighted as:

- Of importance

•. Of major importance

1. Nichols JE, Niles JA, Vega S, Nichols JE, Niles JA, Vega SP, et al. Modeling the lung: Design and development of tissue engineered macro- and micro-physiologic lung models for research use. Exp Biol Med (Maywood). 2014;239:1135-69. This paper contains a good review of 2-D and 3-D lung cell culture systems.

2. Guyot A, Hanrahan JW. ATP release from human airway epithelial cells studied using a capillary cell culture system. J Physiol. 2002;545:199-206. 
3. Kirstein MN, Wieman KM, Williams BW, et al. Short versus continuous gemcitabine treatment of non-small cell lung cancer in an in vitro cell culture bioreactor system. Lung Cancer. 2007;58:196204.

4. Grek CL, Newton DA, Qiu Y, et al. Characterization of alveolar epithelial cells cultured in semipermeable hollow fibers. Exp Lung Res. 2009;35:155-74.

5. Fritsche CS, Simsch O, Weinberg EJ, et al. Pulmonary tissue engineering using dual-compartment polymer scaffolds with integrated vascular tree. Int J Artif Organs. 2009;32:701-10.

6. Huh D, Matthews BD, Mammoto A, et al. Reconstituting organlevel lung functions on a chip. Science. 2010;328:1662-8.

7. Kumar Mahto S, Tenenbaum-Katan J, Sznitman J. Respiratory physiology on a chip. Scientifica (Cairo). 2012;2012:364054. This is a good review on microfluidic devices used to study respiratory physiology.

8. Poon C, Boughton P, Ruys AJ. A dynamic perfusion bioreactor approach for engineering respiratory tissues in-vitro. Conf Proc IEEE Eng Med Biol Soc. 2013;2013:6224-7.

9.• Gilpin SE, Guyette JP, Gonzalez G et al. Perfusion decellularization of human and porcine lungs: Bringing the matrix to clinical scale. J Heart Lung Transplant 2013. This study describes the use of bioreactors for rat and human lung decellularization and recellularization.

10.• Nichols JE, Niles J, Riddle M, et al. Productio $\mathrm{n}$ and assessment of decellularized pig and human lung scaffolds. Tissue Eng Part A. 2013;19:2045-62. This paper describes decellularization of, and cell attachment to, acellular pig and human lungs uisng a prototype (Riddle) bioreactor along with ECM evaluation and several functional assessments.

11. Hoganson DM, Pryor HI, 2nd, Vacanti JP. Tissue Engineering and Organ Structure: A Vascularized Approach to Liver and Lung. Pediatr Res 2008.

12. Hoganson DM, Pryor 2nd HI, Bassett EK, et al. Lung assist device technology with physiologic blood flow developed on a tissue engineered scaffold platform. Lab Chip. 2011;11:700-7.

13. Sreenivasan R, Bassett EK, Hoganson DM, et al. Ultra-thin, gas permeable free-standing and composite membranes for microfluidic lung assist devices. Biomaterials. 2011;32:3883-9.

14. Nalayanda DD, Wang Q, Fulton WB, et al. Engineering an artificial alveolar-capillary membrane: a novel continuously perfused model within microchannels. J Pediatr Surg. 2010;45:45-51.

15. Ling TY, Liu YL, Huang YK, et al. Differentiation of lung stem/progenitor cells into alveolar pneumocytes and induction of angiogenesis within a $3 \mathrm{D}$ gelatin-microbubble scaffold. Biomaterials. 2014;35:5660-9.

16. Rochow N, Manan A, Wu WI, et al. An integrated array of microfluidic oxygenators as a neonatal lung assist device: in vitro characterization and in vivo demonstration. Artif Organs. 2014;38: 856-66. This paper describes the use of a novel microfluidic lungassist device.

17. Miller C, George S, Niklason L. Developing a tissue-engineered model of the human bronchiole. J Tissue Eng Regen Med. 2010;4:619-27.

18. Jungebluth $\mathrm{P}$, Alici E, Baiguera $\mathrm{S}$, et al. Tracheobronchial transplantation with a stem-cell-seeded bioartificial nanocomposite: a proofof-concept study. Lancet. 2011;378:1997-2004.

19. Delaere P, Vranckx J, Verleden G, et al. Tracheal allotransplantation after withdrawal of immunosuppressive therapy. N Engl J Med. 2010;362:138-45.

20.• Delaere PR, Vranckx JJ, Den Hondt M. Tracheal allograft after withdrawal of immunosuppressive therapy. N Engl J Med. 2014;370:1568-70. This paper describes the use of a patient's forearm as an in vivo bioreactor for bioengineering a transplantable trachea.
21. He M, Callanan A. Comparison of methods for whole-organ decellularization in tissue engineering of bioartificial organs. Tissue Eng Part B Rev. 2013;19:194-208. This is a good review comparing different methods used for decellularization.

22. Crapo PM, Gilbert TW, Badylak SF. An overview of tissue and whole organ decellularization processes. Biomaterials. 2011;32: 3233-43.

23. Arenas-Herrera JE, Ko IK, Atala A, Yoo JJ. Decellularization for whole organ bioengineering. Biomed Mater. 2013;8:014106.

24. Petersen TH, Calle EA, Zhao L, et al. Tissue-engineered lungs for in vivo implantation. Science. 2010;329:538-41.

25. Petersen TH, Calle EA, Colehour MB, Niklason LE. Bioreactor for the long-term culture of lung tissue. Cell Transplant. 2011;20:111726.

26. Price AP, England KA, Matson AM, et al. Development of a decellularized lung bioreactor system for bioengineering the lung: the matrix reloaded. Tissue Eng Part A. 2010;16:2581-91.

27. Cortiella J, Niles J, Cantu A et al. Influence of Acellular Natural Lung Matrix on Murine Embryonic Stem Cell Differentiation and Tissue Formation. Tissue Eng Part A 2010.

28.• Bonvillain RW, Scarritt ME, Pashos NC et al. Nonhuman primate lung decellularization and recellularization using a specialized large-organ bioreactor. J Vis Exp 2013:e50825. This study describes how to set up a lung bioreactor, decellularize and reseed primate lungs in a step-by-step visual protocol.

29. Mishra DK, Thrall MJ, Baird BN, et al. Human lung cancer cells grown on acellular rat lung matrix create perfusable tumor nodules. Ann Thorac Surg. 2012;93:1075-81.

30. Ott HC, Clippinger B, Conrad C, et al. Regeneration and orthotopic transplantation of a bioartificial lung. Nat Med. 2010;16:927-33.

31.• Price AP, Godin LM, Domek A et al. Automated Decellularization of Intact, Human-Sized Lungs for Tissue Engineering. Tissue Eng Part C Methods 2014. This paper describes the first completely automated decellularization system that standarizes the preparation of acellular lung scaffolds. Detailed instructions are provided for setting up the bioreactor and valve-manifold. It is also amenable to recellularization in a closed system.

32. Khalpey Z, Qu N, Hemphill C et al. Rapid Porcine Lung Decellularization Utilizing a Novel Organ Regenerative Control Acquisition Bioreactor. ASAIO J 2014.

33. Suki B. Assessing the functional mechanical properties of bioengineered organs with emphasis on the lung. J Cell Physiol. 2014;229:1134-40. This is an excellent review on the techniques used to measure the mechanical properties of tissue and also the parameters that need to be considered when bioengineering the lung.

34. White BM, Santhanam A, Thomas D, et al. Modeling and incorporating cardiac-induced lung tissue motion in a breathing motion model. Med Phys. 2014;41:043501.

35. Wagner DE, Bonenfant NR, Sokocevic D, et al. Three-dimensional scaffolds of acellular human and porcine lungs for high throughput studies of lung disease and regeneration. Biomaterials. 2014;35: 2664-79. This paper describes the development of a synthetic pleural subsitute that can be used to coat small lung segments. This enables a more high throughput system for human lung studies using acellular scafffolds that can be perfused and ventilated.

36. Kandasamy K, Parthasarathi K. Quantifying single microvessel permeability in isolated blood-perfused rat lung preparation. J Vis Exp 2014:e51552. This paper describes the develpment of a method to evaluate lung microvessel permeability in real-time.

37. Lee JW, Fang X, Gupta N, et al. Allogeneic human mesenchymal stem cells for treatment of E. coli endotoxin-induced acute lung injury in the ex vivo perfused human lung. Proc Natl Acad Sci U S A. 2009;106:16357-62.

38. McAuley DF, Curley GF, Hamid UI, et al. Clinical grade allogeneic human mesenchymal stem cells restore alveolar fluid clearance in 
human lungs rejected for transplantation. Am J Physiol Lung Cell Mol Physiol. 2014;306:L809-15. This study describes the ability of MSCs to recondition human lungs ex vivo and the potential for increasing donor lung use.

39. Steen S, Liao Q, Wierup PN, et al. Transplantation of lungs from non-heart-beating donors after functional assessment ex vivo. Ann Thorac Surg. 2003;76:244-52. discussion 252.

40. Nelson K, Bobba C, Ghadiali S, et al. Animal models of ex vivo lung perfusion as a platform for transplantation research. World $\mathrm{J}$ Exp Med. 2014;4:7-15.

41. Cypel M, Yeung JC, Liu M, et al. Normothermic ex vivo lung perfusion in clinical lung transplantation. N Engl J Med. 2011;364:1431-40.

42. Warnecke G, Moradiellos J, Tudorache I, et al. Normothermic perfusion of donor lungs for preservation and assessment with the Organ Care System Lung before bilateral transplantation: a pilot study of 12 patients. Lancet. 2012;380:1851-8.

43. Olmer R, Lange A, Selzer S, et al. Suspension culture of human pluripotent stem cells in controlled, stirred bioreactors. Tissue Eng Part C Methods. 2012;18:772-84.

44. Siti-Ismail N, Samadikuchaksaraei A, Bishop AE, et al. Development of a novel three-dimensional, automatable and integrated bioprocess for the differentiation of embryonic stem cells into pulmonary alveolar cells in a rotating vessel bioreactor system. Tissue Eng Part C Methods. 2012;18:263-72.

45. Herriges M, Morrisey EE. Lung development: orchestrating the generation and regeneration of a complex organ. Development. 2014;141:502-13. This is an excellent review of recent knowledge on lung development.

46. Hogan BL, Barkauskas CE, Chapman HA, et al. Repair and regeneration of the respiratory system: complexity, plasticity, and mechanisms of lung stem cell function. Cell Stem Cell. 2014;15:123-38. This is an excellent review on lung stem cells.

47. Kotton DN, Morrisey EE. Lung regeneration: mechanisms, applications and emerging stem cell populations. Nat Med. 2014;20:
822-32. This is an excellent review on the role of epithelial stem and progenitor cells in lung regeneration.

48.• Treutlein B, Brownfield DG, Wu AR, et al. Reconstructing lineage hierarchies of the distal lung epithelium using single-cell RNA-seq. Nature. 2014;509:371-5. This is the first study to comprehensively define the heterogeneity of the distal lung epithleium using the stateof-the-art RNA-seq technique.

49.• Huang SX, Islam MN, O'Neill J, et al. Efficient generation of lung and airway epithelial cells from human pluripotent stem cells. Nat Biotechnol. 2014;32:84-91. This study describes directed differentiation of human iPS cells into the many different lung epithelial cell types that will be required for lung bioengineering.

50. Ghaedi M, Mendez JJ, Bove PF, et al. Alveolar epithelial differentiation of human induced pluripotent stem cells in a rotating bioreactor. Biomaterials. 2014;35:699-710.

51. Badylak SF, Taylor D, Uygun K. Whole-organ tissue engineering: decellularization and recellularization of three-dimensional matrix scaffolds. Annu Rev Biomed Eng. 2011;13:27-53.

52.• Calle EA, Ghaedi M, Sundaram S, et al. Strategies for whole lung tissue engineering. IEEE Trans Biomed Eng. 2014;61:1482-96. This is an excellent review on many aspects of lung bioengineering.

53. Aguado BA, Mulyasasmita W, Su J, et al. Improving viability of stem cells during syringe needle flow through the design of hydrogel cell carriers. Tissue Eng Part A. 2012;18:806-15.

54. Chen Y, Bloemen V, Impens S, et al. Characterization and optimization of cell seeding in scaffolds by factorial design: quality by design approach for skeletal tissue engineering. Tissue Eng Part C Methods. 2011;17:1211-21.

55. Allen GB, Suratt BT, Rinaldi L, et al. Choosing the frequency of deep inflation in mice: balancing recruitment against ventilatorinduced lung injury. Am J Physiol Lung Cell Mol Physiol. 2006;291:L710-7.

56. Yeung JC, Cypel M, Waddell TK, et al. Update on donor assessment, resuscitation and acceptance criteria, including novel techniques - Non-heart-beating donor lung retrieval and ex vivo donor lung perfusion. Thorac Surg Clin. 2009;19:261-74. 